\title{
Influencia del ácido giberélico y del nitrato de calcio sobre la duración poscosecha de frutos de fresa (Fragaria sp.)
}

\author{
Influence of gibberellin and calcium nitrate \\ on post-harvest conservation of strawberry \\ (Fragaria sp.) fruits
}

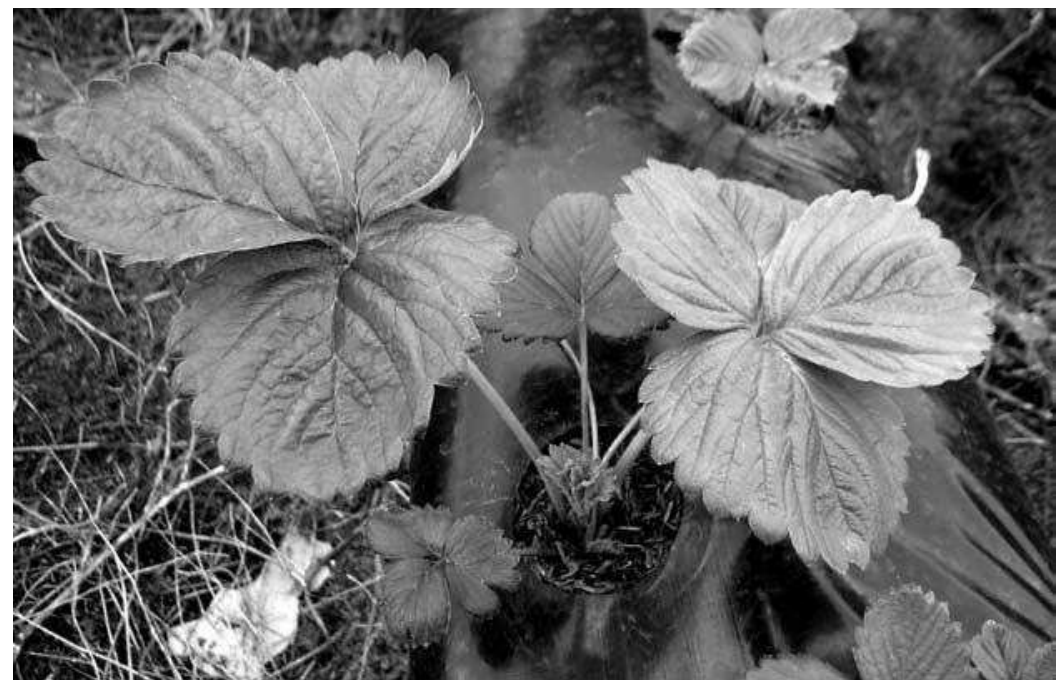

FÁNOR GASIERRA-POSADA1, 2

RICARDO A. SALAMANCA G. ${ }^{1}$

Plantación nueva de fresa

en Arcabuco, Boyacá (Colombia).

Foto: F. Casierra-Posada

\section{RESUMEN}

Los factores precosecha, como el material genético, las condiciones ambientales durante el cultivo, la nutrición y el manejo de cultivo tienen una marcada influencia sobre el comportamiento poscosecha de frutas, especialmente debido a su carácter perecedero. Este trabajo se realizó en un cultivo a campo abierto en Tuta, Colombia, con plantas de fresa de los cultivares Chandler y Sweet Charlie. Se asperjaron 15 días luego de la antesis y al momento de la cosecha con diferentes concentraciones de ácido giberélico y de nitrato de calcio, con miras a determinar su efecto sobre la pérdida de peso y la vida en poscosecha de los frutos. Los tratamientos comprendieron un testigo sin aplicación y 500, 1.500 y $3.000 \mathrm{mg} \mathrm{L}^{-1}$ de ácido giberélico ó 50,150 y $200 \mathrm{mg}$ $\mathrm{L}^{-1}$ de nitrato de calcio aplicados a las hojas y frutos de las plantas. Al momento de la cosecha se realizaron pruebas de calidad en la determinación de sólidos solubles totales, acidez titulable y peso del fruto. En otros frutos se determinó la pérdida diaria, como la pérdida máxima de peso. Los resultados no mostraron ningún efecto de los productos aplicados o de sus concentraciones sobre la calidad del fruto. Los frutos de 'Chandler' se conservaron por mayor tiempo en poscosecha que los de 'Sweet Charlie'. Los frutos tratados con nitrato de calcio duraron 16,4\% más tiempo, mientras que los aplicados con ácido giberélico duraron $11,6 \%$ más que los testigos. La pérdida de peso fue proporcional a la duración en poscosecha. Comparando los resultados, se observó una respuesta de los frutos a los tratamientos, fuertemente dependiente del cultivar.

1 Grupo de Investigación Ecofisiología Vegetal, Facultad de Ciencias Agropecuarias, Universidad Pedagógica y Tecnológica de Colombia (UPTC), Tunja (Colombia).

2 Autor para correspondencia. fanor.casierra@uptc.edu.co 


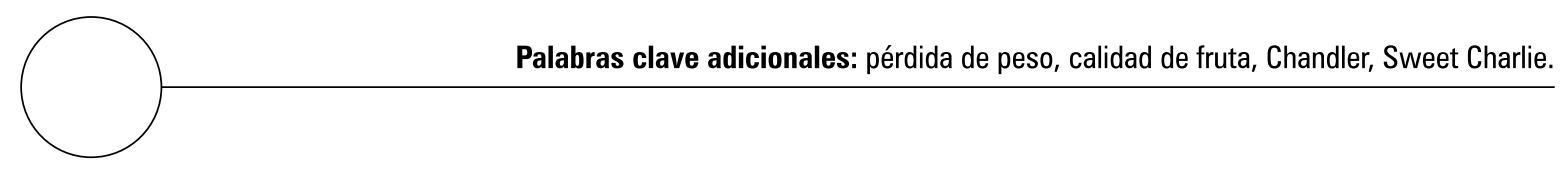

\section{ABSTRACT}

Pre-harvest factors, such as genetic background, environmental conditions during cultivation, nutrition and crop handling, noticeably influence the post-harvest condition of perishable fruits. This study was carried out in an open field crop of strawberries in Tuta, Colombia to evaluate effects of gibberellin and calcium nitrate on both weight loss and fruit shelf life. Leaves and fruits of 'Chandler' and 'Sweet Charlie' plants were sprayed with 500, 1,500 and 3,000 ppm gibberellin and 50, 150 and 200 ppm calcium nitrate 15 days after anthesis and at harvest. Total fruit soluble solids, titratable acidity and fruit fresh weight were evaluated post-harvest and daily weight loss and maximal post-harvest life were assessed for each treatment. The results showed no effect of the sprayed products on fruit quality. However, the fruits of cv. Chandler lasted longer during post-harvest than those of Sweet Charlie. Also, fruits treated with calcium nitrate lasted $16,4 \%$ longer, while gibberellin solutions prolonged their post-harvest life only $11,6 \%$ in comparison to control fruits. Post-harvest weight loss was directly proportional to post-harvest time. The response of fruit to treatments was strongly dependent on the cultivar.

Additional keywords: weight loss, fruit quality, Chandler, Sweet Charlie.

Fecha de recepción: 25-03-2008

Aprobado para publicación: 03-06-2008
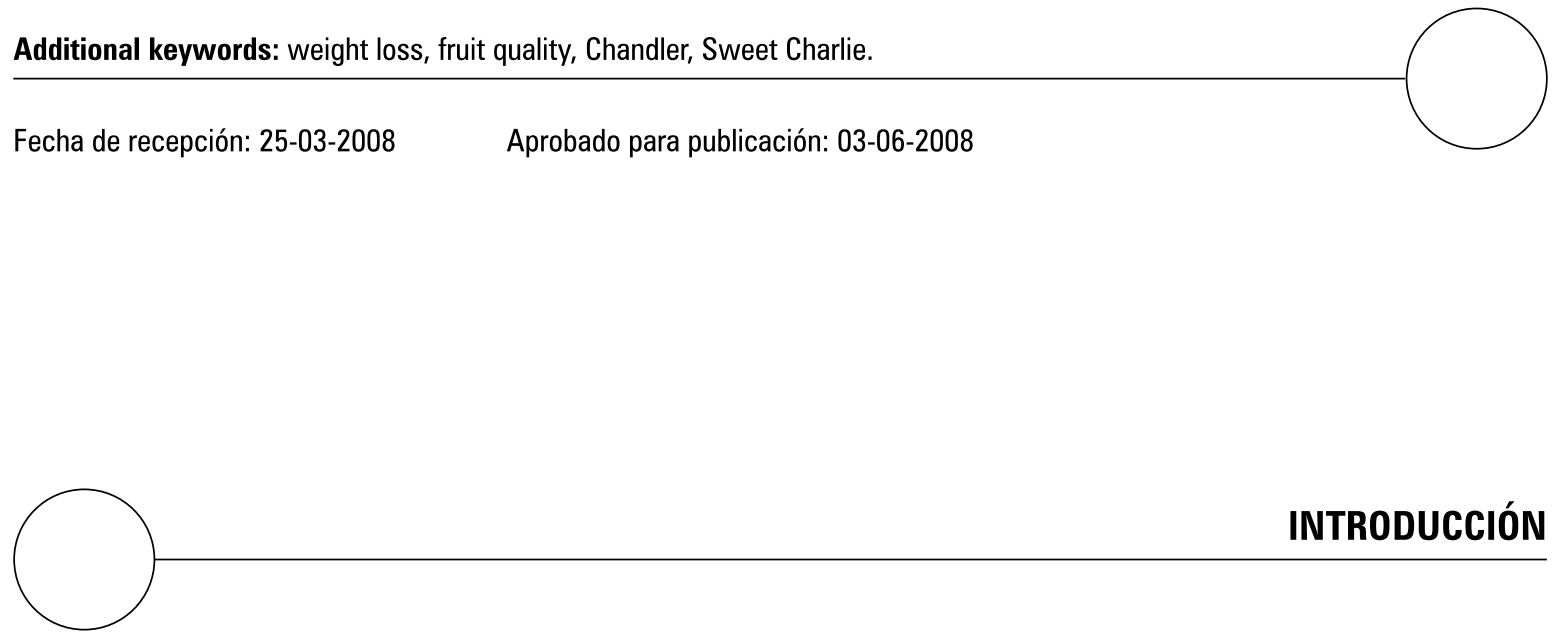

A diferencia de los países de la zona templada, donde la producción se limita al verano, en Colombia es posible la producción de fresa (Fragaria sp.) durante todo el año, con oferta alta de noviembre a febrero y junio a septiembre y oferta media en octubre y de marzo a mayo (Corabastos, 2004). En 2006, se cultivaron en Colombia 703 ha de fresa en los departamentos más productores, Cundinamarca, Antioquia y Cauca, con un rendimiento promedio de $24 \mathrm{t} \mathrm{ha}^{-1}$ a nivel nacional (Agronet, 2008).
La fresa representa un cultivo de alto valor con una vida poscosecha bastante corta, que requiere de tratamientos de poscosecha para prolongar su vida útil (Esmel et al., 2004). El color, el tamaño, la firmeza, la acidez y el sabor dulce hacen de la fresa una de las frutas más populares durante la primavera y el verano en países de zona templada. A pesar de esto, algunos consumidores se sienten insatisfechos con su sabor y con la calidad de algunos tipos de fresa (Azodanlou et al., 2003). Por este motivo, se han realizado ensayos, en los 
cuales, mediante la aplicación en precosecha de fuentes de calcio y reguladores del crecimiento, se busca mejorar la calidad de los frutos. Se han utilizado muchos reguladores de crecimiento con el fin de incrementar el tamaño de la fruta (Guardiola y García-Luis, 2000; Stern et al., 2007). La eficiencia de estos productos es fácilmente evaluable con respecto al tamaño de la fruta, pero es necesario conocer su influencia en las características bioquímicas y de calidad en la misma.

El papel de las auxinas sobre el desarrollo de frutos de fresa está ampliamente reportado. Las auxinas son las responsables del crecimiento del receptáculo floral y, por tanto, del incremento del tamaño de los frutos. En fresa, Nitsch (1950) demostró que los compuestos hormonales producidos durante el desarrollo de las semillas estaban asociados con el crecimiento del fruto.

Se reportan algunos efectos positivos de las aplicaciones de ácido giberélico sobre la producción de fruta, el acortamiento del periodo entre la siembra y la primera fructificación, el número de frutos y la duración del periodo de cosecha; sin embargo, esta hormona también puede reducir el peso de la fruta (Singh et al., 1960; Tehranifar y Battey, 1997). Choma y Himelrick (1984) observaron que las aplicaciones de ácido giberélico a cultivares con diferente sensibilidad al fotoperiodo incrementaban el peso y número de frutos. A pesar de esto, se puede esperar una respuesta contraria de las plantas cuando se usan dosis muy altas de la hormona (Dennis y Bennett, 1969; Weidman y Stang, 1983; Tehranifar y Battey, 1997).

El calcio se aplica comúnmente en varios cultivos frutícolas con miras a mantener o incrementar la calidad de los frutos. Se ha aplicado a fresas del cultivar Sweet Charlie tiosulfato de calcio para lograr un incremento en la vida poscosecha de los frutos (Esmel et al., 2004). El calcio ha sido reportado ampliamente como un elemento esencial que desempeña un papel importante en el mantenimiento de la calidad poscosecha en cul- tivos hortofrutícolas. El efecto del calcio sobre el comportamiento poscosecha de productos agrícolas se atribuye, en particular, a su función como estabilizador de la membrana celular (Kirkby y Pilbeam, 1984).

El objetivo del presente trabajo fue evaluar el efecto de la aplicación de diferentes dosis de ácido giberélico y de nitrato de calcio, realizadas en la mitad del periodo de desarrollo de los frutos y al momento de la cosecha, sobre la vida poscosecha de frutos de dos cultivares de fresa.

\section{MATERIALES Y MÉTODOS}

Este ensayo se realizó en Tuta, Colombia, población ampliamente reconocida por ser productora de fresa, ubicada a $05^{\circ} 41^{\prime} 36^{\prime \prime} \mathrm{N}, 73^{\circ} 13^{\prime} 51^{\prime \prime} \mathrm{W}$ y $2.600 \mathrm{~m}$ de altura sobre el nivel del mar. La temperatura media es de $14^{\circ} \mathrm{C}$ y la precipitación anual de $935 \mathrm{~mm}$. Como material vegetal se utilizaron plantas de fresa (Fragaria sp.) de 18 meses de los cultivares Chandler y Sweet Charlie, dado que son cultivares comúnmente sembrados en la zona. Las plantas fueron seleccionadas, rotuladas y se les eliminó la fructificación existente al iniciar el ensayo. Al inicio del trabajo se marcaron 150 flores en diferentes plantas y los tratamientos respectivos se realizaron a los 15 días después de la antesis y al momento de la cosecha. Estas fechas se determinaron basadas en que en la región los frutos duran 28-30 días desde la apertura de la flor hasta el punto de cosecha. Los tratamientos consistieron en la aplicación foliar de las soluciones acuáticas del ácido (Progibb $^{\circledR}$, Bayer CropScience) en concentraciones de 0, 500, 1.500 ó $3.000 \mathrm{mg} \mathrm{L}^{-1}$ y nitrato de calcio $\left(\mathrm{Ca}\left(\mathrm{NO}_{3}\right)_{2} 4 \mathrm{H}_{2} \mathrm{O}\right.$, Merck) en concentraciones de 0, 50, 150 ó $200 \mathrm{mg} \mathrm{L}^{-1}$.

Al momento de la cosecha se realizaron las pruebas de calidad en 50 frutos con madurez de cosecha (fruto con el $80 \%$ de color rojo), que consistieron en determinar los sólidos solubles totales ('Brix), la acidez total titulable y el peso fresco 
del fruto. Los frutos restantes se llevaron al Laboratorio de Fisiología Vegetal de la Universidad Pedagógica y Tecnológica de Colombia (Tunja) $y$ se mantuvieron en un ambiente a temperatura promedio del $15 \pm 2^{\circ} \mathrm{C}$ y humedad relativa de $71 \% \pm 5 \%$ hasta que los frutos mostraran síntomas de senectud y perdieran por completo su calidad. Para este estudio se tomaron 100 frutos por cultivar y por tratamiento; a diario se pesó cada fruto de manera individual, para determinar su pérdida de peso fresco. El trabajo consistió en un ensayo factorial de $2^{*} 2^{*} 3$, cuyo primer factor fue el cultivar (Chandler o Sweet Charlie), el segundo factor, los dos productos (ácido giberélico o nitrato de calcio) y el tercer factor las tres concentraciones (baja, media, alta) de los productos. Dada la naturaleza diferente de los dos productos utilizados, se dosificó de acuerdo con cada uno de estos; por tanto, para facilitar el acceso de los datos al programa estadístico, se utilizó el concepto de dosis baja, media, alta, las cuales correspondían a 0,500, 1.500 y 3.000 $\mathrm{mg} \mathrm{L}^{-1}$ de ácido giberélico y 50, 150 y $200 \mathrm{mg}$ $\mathrm{L}^{-1}$ de nitrato de calcio, respectivamente. Los resultados se analizaron mediante un análisis de varianza (Anova) y la prueba de separación de promedios de Tukey $(P \leq 0,05)$. El análisis estadístico se realizó mediante la aplicación de SPSS, versión 11.5.1.

\section{RESULTADOS Y DISCUSIÓN}

El efecto de los tratamientos implementados sobre la calidad del fruto se evaluó en relación con el contenido de sólidos solubles totales y con la acidez total titulable, en la cual, según el análisis de varianza, no se encontraron diferencias significativas para ninguna de las variables evaluadas. A pesar de esto, vale la pena destacar que para 'Chandler' y 'Sweet Charlie' se encontraron valores para los sólidos solubles totales correspondientes a 8,02 y $8,92^{\circ} \mathrm{Brix}$, respectivamente; mientras que en el caso de la acidez total titulable, Chandler presentó 0,09\% y Sweet Charlie1,10\%. De igual manera, Roussos et al. (2008) no encontra- ron un impacto significativo de la aplicación de reguladores del crecimiento sobre estos mismos parámetros en fresa, así como tampoco hubo cambios importantes en el contenido de carbohidratos, ni en el color de la fruta, a pesar del incremento en la concentración de antocianinas como consecuencia de los tratamientos. Adicionalmente, los mismos autores reportaron que la actividad antioxidante del jugo de los frutos fue ligeramente mayor en los frutos provenientes de las plantas control, lo que podría ser el resultado de un mayor contenido de fenoles, o-difenoles, flavonoides y flavanoles. Estos resultados también han sido reportados por Ozguven y Yilmaz (2002), quienes no encontraron cambios en la acidez titulable en frutos de fresa luego de una aplicación de ácido giberélico. De hecho, el efecto del ácido giberélico sobre las características fisicoquímicas de los frutos de fresa son altamente dependientes del cultivar, de la dosis aplicada y de la sensibilidad de las plantas al fotoperiodo, como reporta Kirschbaum (1998), quien presenta un listado de características de las plantas y frutos de fresa que fueron afectados por aplicaciones de ácido giberélico en precosecha.

Por otro lado, en diferentes reportes se menciona que la aplicación de reguladoras del crecimiento a las plantas tiene un efecto directo sobre el tamaño de fruto sin afectar el contenido de sólidos solubles ni la acidez titulable, tanto en fresa (Rohloff et al., 2002) como en otras especies (Amoros et al., 2004; Stern et al., 2007). Sin embargo, este resultado no se evidenció en el presente trabajo porque no hubo respuesta de los frutos a las aplicaciones, en cuanto al incremento en tamaño. Sólo se encontró que los frutos del cultivar Chandler fueron 40,7\% más pesados que los de Sweet Charlie, pero este es un parámetro resultante del material vegetal utilizado y no por defecto de los productos aplicados.

Se presentó un efecto positivo de los tratamientos implementados sobre la prolongación de la vida en poscosecha de los frutos. Al respecto, hubo diferencias altamente significativas $(P \leq 0,01)$ del culti- 
var. El cultivar Chandler mostró una duración de 18,4\% más tiempo en poscosecha que el cultivar Sweet Charlie. La interacción producto y concentración fue significativa $(P \leq 0,05)$. Los frutos tratados con ácido giberélico duraron $11,6 \%$ más tiempo en referencia con los frutos de las plantas control, mientras aquellos tratados con nitrato de calcio tuvieron $16,4 \%$ más tiempo en poscosecha, en comparación con los controles. En cuanto al $\mathrm{AG}_{3}$, en concentración ascendente, los frutos duraron 14,8; 10,3 y 17,0\% más tiempo que los frutos de las plantas control. En lo relacionado con la significancia de la interacción de los tres factores, sólo se presentó diferencia altamente significativa para la interacción cultivar * producto $(P \leq 0,01)$.

El calcio contribuye a unir las sustancias pécticas en las paredes celulares (Demarty et al., 1984). Las implicaciones poscosecha de un buen suministro de calcio radican en que altos contenidos de calcio en los frutos causan una reducción en la tasa de maduración, respiración, producción de etileno y ablandamiento de los frutos (Ferguson, 1984). El calcio se absorbe principalmente a través de los tejidos jóvenes de los ápices radicales y se transloca a través del torrente de transpiración (Mengel et al., 2001; por tanto, aplicaciones precosecha con productos al suelo que contengan calcio tienen como consecuencia un mejoramiento en el comportamiento poscosecha y en la calidad de frutos en fresa. Sin embargo, en el presente ensayo, las aplicaciones se realizaron vía foliar, con lo cual fue también posible mejorar parcialmente su comportamiento en poscosecha, a pesar de la condición de inmovilidad del elemento cuando es aplicado a los tejidos aéreos, como lo corroboraron Eaves y Leefe (1962). Igualmente, Cheour et al. (1990; 1991) encontraron que aplicaciones de cloruro de calcio reducen la maduración y el desarrollo de hongos en poscosecha e incrementan la firmeza del fruto durante la cosecha y la poscosecha.

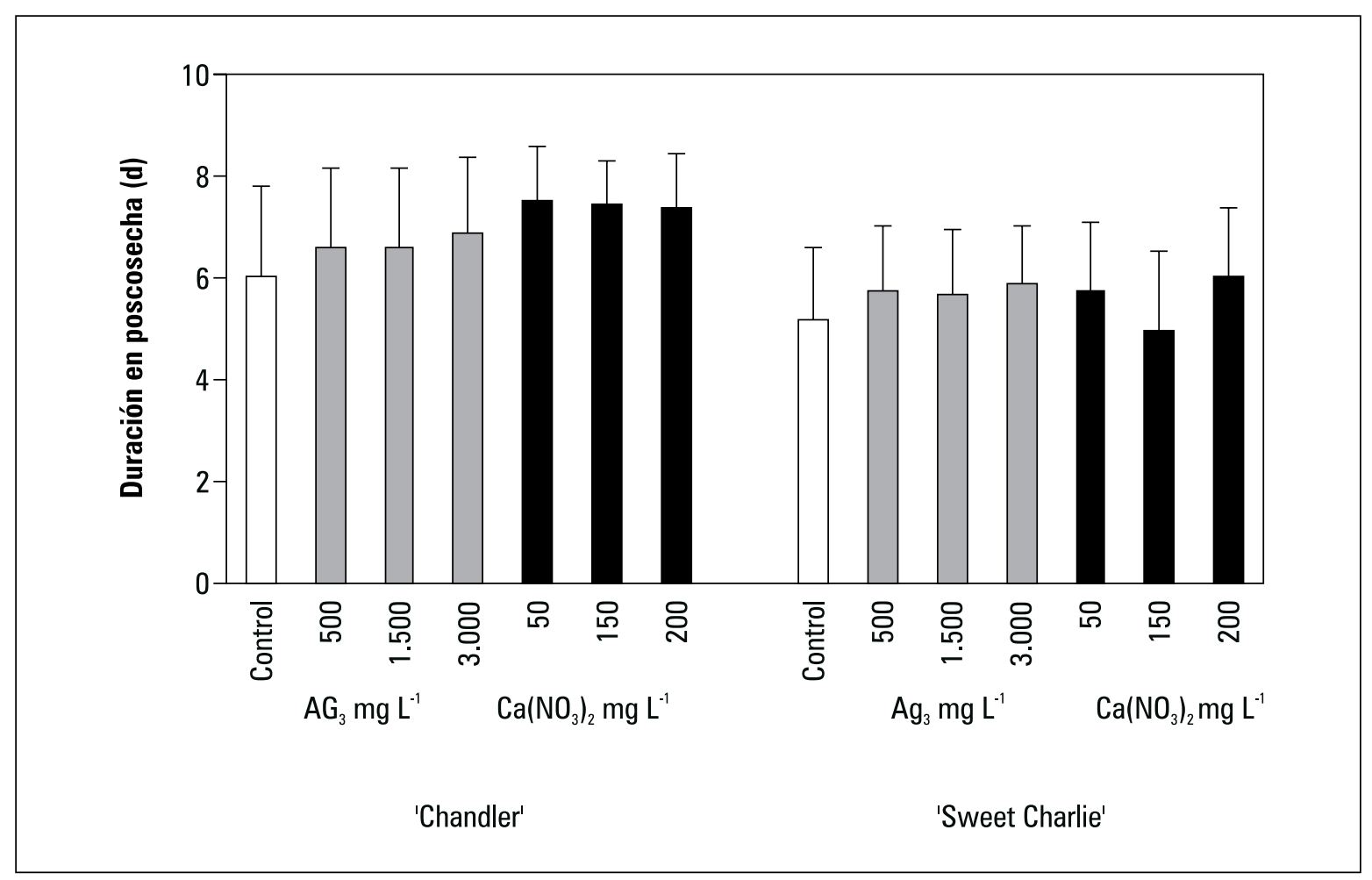

Figura 1: Efecto de la aplicación de ácido giberélico y de nitrato de calcio sobre la duración de frutos de fresa (Fragaria sp.) en poscosecha. Las barras sobre las columnas indican la desviación estándar. 
Por otro lado, se encontró un efecto favorable de aplicaciones foliares de calcio sobre la calidad de la fruta, pero no hubo efecto significativo sobre la producción de fruta o sobre la reducción de frutos deformados (Wojcik y Lewandouski, 2003). En contraste, Erincik et al. (1998), en ensayos realizados tanto en campo como en invernadero, reportaron que no hubo diferencias significativas de las aplicaciones foliares de cloruro de calcio sobre el contenido de $\mathrm{Ca}$ en los frutos de fresa, ni tampoco sobre la producción de frutos, firmeza, sólidos solubles, acidez y color externo de los frutos. Makus y Morris (1989; 1998) sustentan la conclusión que aplicaciones tanto foliares como edáficas de calcio no incrementan la calidad de frutos de fresa ni la distribución del elemento dentro de los frutos. Por tanto, se planteó la importancia de la fuente de calcio y fue así, como Esmel et al. (2004) utilizaron tiosulfato de calcio por medio de fertirrigación en plantas de fresa, sin encontrar significancia en la calidad de frutos. Todas estas apreciaciones conducen a concluir que hay un componente genético involucrado en los resultados, dado que los investigadores mencionados realizaron sus ensayos utilizando diferentes cultivares de fresa. Este aspecto se evidenció de igual manera en el presente trabajo, como se discutirá posteriormente.

Los resultados del ensayo mostraron diferencias altamente significativas de los factores cultivar, producto y concentración sobre la pérdida de peso en poscosecha, pero no se presentó diferencia para las interacciones entre los factores $(P \leq 0,01)$. Los frutos del cultivar Chandler perdieron 5,4\% más peso que los de Sweet Charlie. A su vez, los frutos de plantas tratadas con nitrato de calcio en precosecha perdieron en promedio $23,64 \%$ más peso que aquellos tratados con ácido giberélico, en los que el promedio de pérdida fue de $3,8 \%$, en relación con el control sin aplicación. Adicionalmente, se pudo constatar una pérdida de peso

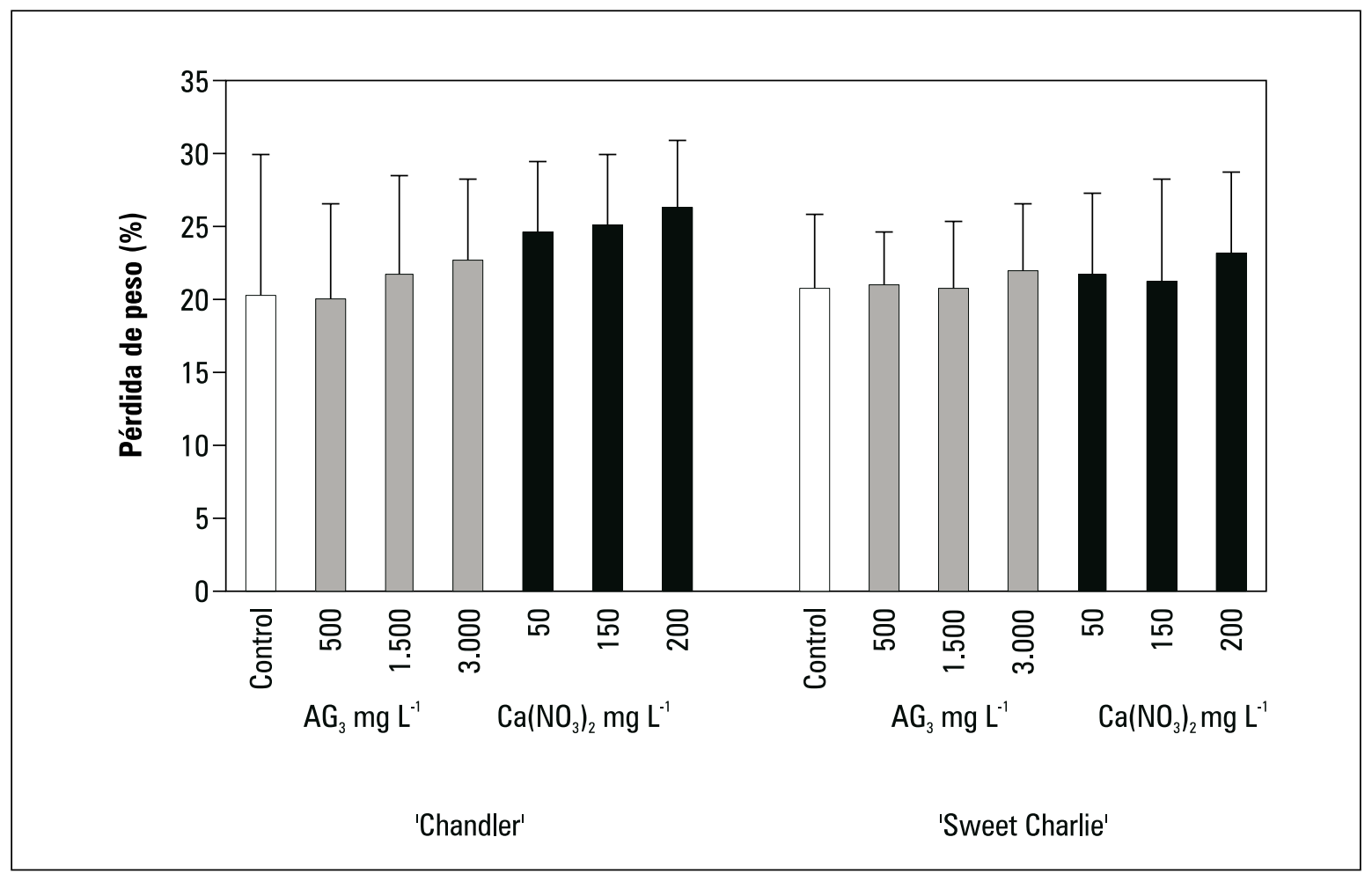

Figura 2: Efecto de la aplicación de ácido giberélico y de nitrato de calcio sobre pérdida de peso en frutos de fresa (Fragaria sp.) en poscosecha. Las barras sobre las columnas indican la desviación estándar. 
de frutos de manera proporcional al incremento en la concentración del producto aplicado en precosecha. Las dosis inferior, media y alta indujeron 5,$9 ; 8,0$ y $14,5 \%$ de pérdida de peso en la poscosecha de frutos en relación con los frutos de las plantas control.

De hecho, la mayor pérdida de peso encontrada en los frutos, como consecuencia de la aplicación de nitrato de calcio, fue resultado de que los frutos tratados con este compuesto duraron más tiempo en poscosecha; por tanto, la exposición de sus epidermis a las condiciones ambientales que les inducían la perdida de peso fue más prolongada. Las pérdidas de peso de los frutos de fresa durante la poscosecha son la consecuencia de la respiración de los frutos (California Strawberry Commission, 2007) y también de las pérdidas de agua (Keutgen y Pawelzik, 2008). A pesar de estas modificaciones encontradas en poscosecha, estos últimos autores no encontraron cambios en el contenido relativo de materia seca. La pérdida de peso estaba acompañada por una apariencia y cambios de color poco atractivos de los frutos hacia el final de la vida en poscosecha, lo cual se evidenció claramente en el presente ensayo, en el cual los frutos adquirían una falta de brillo y se tornaban de una coloración más oscura en la medida en que perdían humedad, resultados que también fueron reportados por Nunes et al. (1995) y Knee y Aggrawal (2000).

Los resultados del presente ensayo estuvieron influenciados por el componente genético. Fue así, como los dos cultivares utilizados respondieron de manera diferente a las aplicaciones del ácido giberélico y de nitrato de calcio. La figura 3 muestra que en conjunto los frutos del cultivar Chandler duraron un día más en poscosecha que los de Sweet Charlie, independientemente del producto aplicado o de su concentración, lo cual pone de manifiesto que la respuesta de los frutos de fresa a tratamientos orientados al mejoramiento de la calidad o a la prolongación de la vida poscosecha es fuertemente dependiente del material vegetal utilizado en los ensayos. Esto fue corroborado por diferentes investigadores citados anteriormente quienes encontraron respuestas contradictorias o inconsistentes de diversos cultivares a las aplicaciones de diferentes fuentes de calcio, en relación con la producción y calidad del fruto.

Adicionalmente, se reporta que los frutos de fresa pierden su posibilidad de mercadeo con una pérdida de humedad del orden de 6\% (Nunes et al., 1995), lo cual fue alcanzado en todos los tratamientos, casi desde el primer día de almacenamiento. Evaluaciones similares en cuanto al comportamiento de frutos en poscosecha, en respuesta a tratamientos previos, arrojaron resultados similares. Es así como Keutgen y Pawelzik (2008) evaluaron el comportamiento de frutos de fresa expuestos a ozono y encontraron que en todos los tratamientos, los frutos alcanzaron el $6 \%$ de pérdida de humedad al quinto día luego de la cosecha, adicionalmente, que los tratamientos con ozono, durante el crecimiento, reducen las pérdidas de peso fresco en $1 \%$, lo cual es un valor pequeño que no incide de manera considerable en las posibilidades de mercado de los frutos, debido a los cambios en la apariencia de los mismos.

Debe hacerse énfasis en que uno de los objetivos del presente estudio era la evaluación de los tratamientos en cuanto a la calidad y duración de los frutos en poscosecha, sin condiciones especiales de almacenamiento que pudieran intervenir con la pérdida de humedad, de esta manera, los frutos expuestos a condiciones extremas de almacenamiento, tendrían la posibilidad de expresar su durabilidad afectada por los productos aplicados en precosecha. En concordancia con los resultados del presente estudio, se reporta que la vida poscosecha de los frutos se ve ampliamente afectada por factores como la variedad, el sitio cultivo, el comportamiento climático durante la temporada, las prácticas precosecha y las condiciones ambientales en poscosecha (California Strawberry Commission, 2007). 

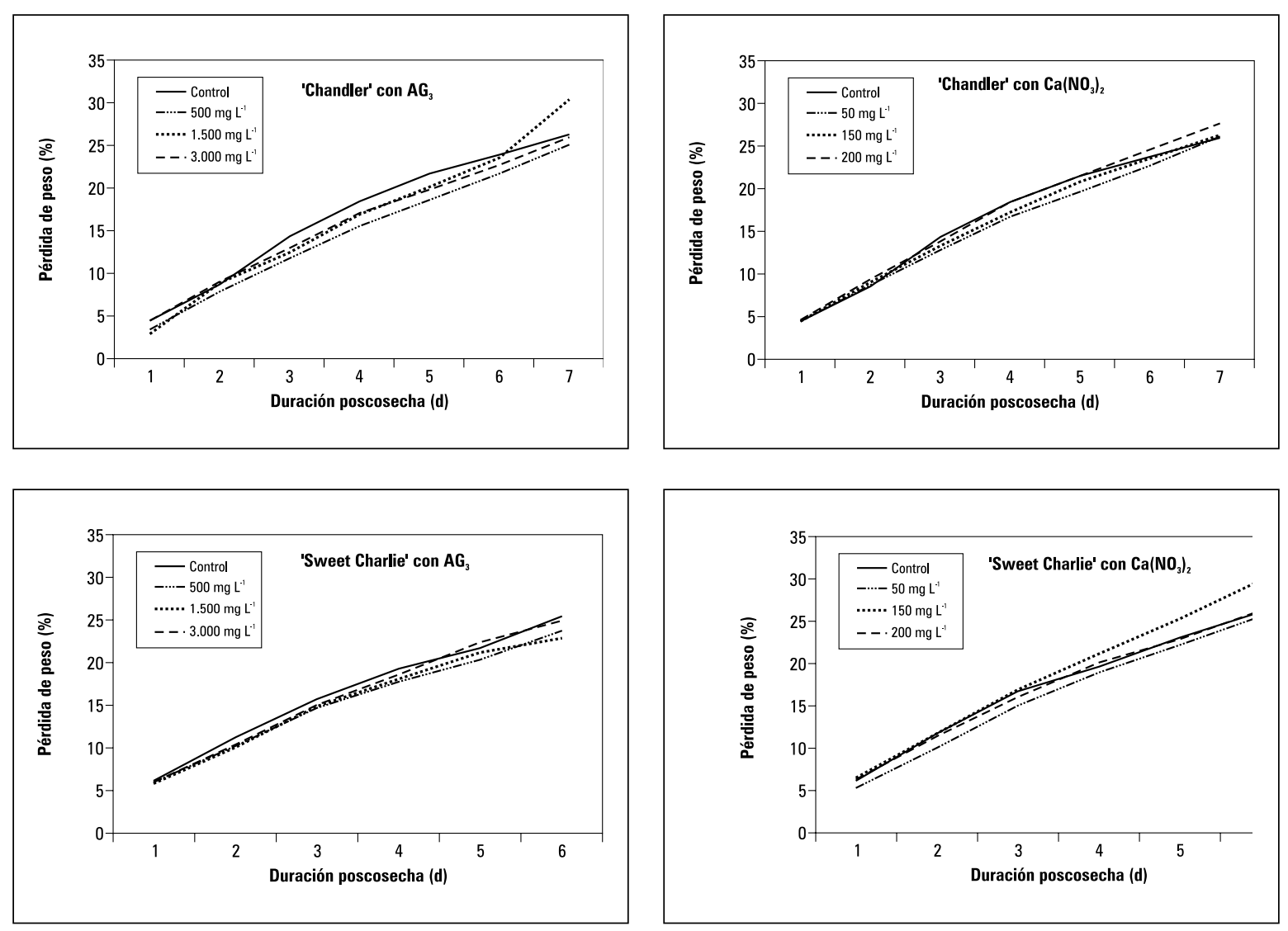

Figura 3. Porcentaje de pérdida de peso fresco en poscosecha en frutos de fresa (Fragaria sp.) de los cultivares Chandler y Sweet Charlie luego de la aplicación de ácido giberélico y de nitrato de calcio en diferentes concentraciones.

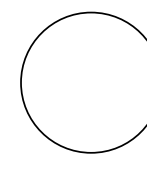

AGRADECIMIENTOS

Este estudio fue desarrollado con el apoyo de la Dirección de Investigaciones (DIN) de la Universidad Pedagógica y Tecnológica de Colombia, Tunja, en el marco del plan de trabajo del Grupo de Investigación Ecofisiología Vegetal, adscrito al programa de Ingeniería Agronómica de la Facultad de Ciencias Agropecuarias. 


\section{REFERENCIAS BIBLIOGRÁFICAS}

Agronet. 2008. Área cosechada, producción y rendimiento de fresa 1992-2006. En: http://www.agronet. gov.co/; consulta: 21 de mayo de 2008.

Amoros, A.; P. Zapata; M.T. Pretel; M.A. Botella; M.S. Almansa y M. Serrano. 2004. Role of naphthalene acetic acid and phenothiol treatments on increasing fruit size and advancing fruit maturity in loquat. Scientia Hort. 101, 387-398.

Azodanlou, R.; C. Darbellay; J.L. Luisier; J.C. Villettaz y R. Amado. 2003. Quality assessment of strawberries (Fragaria species). J. Agric. Food Chem. 51, 715-721.

California Strawberry Commission. 2007. Best handling practices for fresh strawberries. En: http://www. calstrawberry.com/fileData/docs/Best_Handling Practice_for_Fresh_Strawberries_-_Final_03_12_ 07.pdf; consülta: 5 de marzo de $200 \overline{8}$.

Cheour, F.; C. Willemot; J. Arul; Y. Desjardins; J. Makhlout; P.M. Charest y A. Gosselin. 1990. Foliar application of calcium chloride delays post-harvest ripening of strawberry. J. Amer. Soc. Hort. Sci. 115, 789-792.

Cheour, F.; C. Willemot; J. Arul; J. Makhlout y Y. Desjardins. 1991. Post-harvest response of two strawberry cultivars to foliar applications of calcium chloride. HortScience 26, 1186-1188.

Choma, M.E. y D.G. Himelrick. 1984. Responses of dayneutral, June-bearing and everbearing strawberry cultivars to gibberellic acid and phthalamide treatments. Scientia Hort. 22, 257-264.

Corabastos. 2004. Fresa. Portal de Frutas y Hortalizas de Colombia para el Mundo. En: http://frutasyhortalizas.com.co/portal/includej/product_view.php; consulta: 9 de mayo de 2008.

Demarty, M.; C. Morvan y M. Thellier. 1984. Calcium and the cell wall. Plant Cell Environ. 7, 441-448.

Dennis, F.G. y H.O. Bennett. 1969. Effect of gibberellic acid and deflowering upon runner and inflorescence development in an everbearing strawberry. J. Amer. Soc. Hort. Sci. 94, 534-537.

Eaves, C.A. y J.S. Leefe. 1962. Note on the influence of calcium on the firmness of strawberries. Can. J. Plant Sci. 42, 746-747.
Erincik, O.; L.V. Madden; J.C. Scheerens y M.A. Ellis. 1998. Evaluation of foliar applications of calcium chloride for control of Botrytis fruit rot on strawberry and effects on strawberry fruit quality. Adv. Strawberry Res. 17, 7-13.

Esmel, C.E.; J.R. Duval y A.A. Steven. 2004. The influence of calcium thiosulfate on yieldand postharvest quality of 'Sweet Charlie' strawberry. Proc. Fla. State Hort. Soc. 117, 48-51.

Ferguson, I. 1984. Calcium in plant senescence and fruit ripening. Plant Cell Environ. 7, 477-489.

Guardiola, J.L. y A. Garcia-Luis. 2000. Increasing fruit size in citrus. Thinning and stimulation of fruit growth. Plant Growth Regul. 31, 121-132.

Keutgen, A. y E. Pawelzik. 2008. Influence of pre-harvest ozone exposure on quality of strawberry fruit under simulated retail conditions. Postharv. Biol. Technol. 49, 10-18.

Kirkby, E.A. y D.J. Pilbeam. 1984. Calcium as a plant nutrient. Plant Cell Environ. 7, 397-405.

Kirschbaum, D.S. 1998. Temperature and growth regulator effects on growth and development of strawberry (Fragaria x ananassa Duch.). Master Sc. thesis. University of Florida, Gainesville, FL.

Knee, M. y D. Aggrawal. 2000. Evaluation of vacuum containers for consumer storage of fruits and vegetables. Postharv. Biol. Technol. 19, 55-60.

Makus, D.J. y J.R. Morris. 1989. Influence of soil and foliar applied calcium on strawberry fruit nutrient and postharvest quality. Acta Hort. 265, 443-446.

Makus, D.J. y J.R. Morris. 1998. Preharvest calcium applications have little effect on mineral distribution in ripe strawberry fruit. HortScience 33, 64-66.

Mengel, K., E.A. Kirkby, H. Kosegarten y T. Appel. 2001. Principles of plant nutrition. $5^{\text {th }}$ ed. Kluwer Academic Publishers, Dordrecht, The Netherlands.

Nitsch, J.P. 1950. Growth and morphogenesis of the strawberry as related to auxin. Amer. J. Bot. 37, 211-215. 
Nunes, M.C.N.; J.K. Brecht; A.M.M.B Morais y S.A. Sargent. 1995. Physical and chemical quality characteristics of strawberries after storage are reduced by a short delay to cooling. Postharv. Biol. Technol. $6,17-28$.

Ozguven, A.I. y C. Yilmaz. 2002. The effect of gibberellic acid treatments on the yield and quality of strawberry (Fragaria $x$ ananassa) cv. Camarosa. Acta Hort. 567, 277-280.

Rohloff, J.; S. Fiskaa H. y T. Iversen. 2002. The effect of plant biochemical regulators on strawberry production in field trials under drip irrigation management at 5 locations in Norway. Acta Hort. 567, 463-466.

Roussos P.A.; N.-K. Denaxa y T. Damvakaris. 2008. Strawberry fruit quality attributes after application of plant growth stimulating compounds. Scientia Hort. 119(2), 138-146.
Singh, J.P.; G.S. Randhawa y N.L. Jain. 1960. Response of strawberry to gibberellic acid. Indian J. Hort. $17,21-30$

Stern, R.A.; M.A. Flaishman y R. Ben-Arie. 2007. Effect of synthetic auxins on fruit size of five cultivars of Japanese plum (Prunus salicina Lindl.). Scientia Hort. 112, 304-309.

Tehranifar, A. y N.H. Battey. 1997. Comparison of the effects of $\mathrm{GA}_{3}$ and chilling on vegetative vigour and fruit set in strawberry. Acta Hort. 439, 627-631.

Weidman, R.W. y J. Stang. 1983. Effects of gibberellins $\left(\mathrm{GA}_{4+7}\right)$, 6-benzyladenine (6-BA) and promalin $\left(\mathrm{GA}_{4+7}+6-\mathrm{BA}\right)$ plant growth regulators on plant growth, branch crown and flower development in 'Scott' and 'Raritan' strawberries. Adv. Strawberry Prod. 2, 15-17.

Wojcik, P. y M. Lewandowski. 2003. Effect of calcium and boron sprays on yield and quality of 'Elsanta' strawberry. J. Plant Nutr. 26, 671-682. 